\title{
A Novel Parameterisation of Phase Plots for Monitoring of Parkinson's Disease.
}

\author{
Michael Dunne-Willows, Member, IEEE, Paul Watson, Jian Shi, Lynn Rochester, Silvia Del Din, Member, IEEE.
}

\begin{abstract}
Parkinson's Disease (PD) can lead to impaired/slowed movement, gait impairments and increased risk of falling. Wearable technology-based gait analysis is emerging as a powerful tool to detect early disease and monitor progression. Here we present a novel approach to producing an objective, compact and personalised overview of a patients' gait pattern. Phase plots were constructed in 41 people with $P D$ and 38 controls (CL) from accelerometry data collected during straight intermittent walks with a single triaxial accelerometer placed on the lower back. Phase plots were analysed using bivariate Gaussian mixture models and classified based on several apparent features derived from the parameters of said model. Significant differences in phase plot form were found between and PD and CL subjects; with a very high within-subject consistency (reproducibility) (p < 0.0001). PD and CL subjects differ in the types of phase plots produced $(p<0.001)$. Strong connections between spatio-temporal (ST) gait characteristics and phase plot types were found. The presented novel methodology not only showed to be sensitive to pathology (PD vs CL), but can quickly produce a unique fingerprint of a person's gait. This work presents encouraging results for clinical application of an objective, personalised gait feature for disease monitoring and clinical applications.
\end{abstract}

\section{INTRODUCTION}

Parkinson's disease (PD) is the second most common neurological disorder; due to its physical manifestations and degenerative nature, PD can lead to impaired/slowed research movement, gait impairments and increased risk of falling. PD can progress more rapidly if not treated correctly hence the

\footnotetext{
M. Dunne-Willows \& P. Watson are with The EPSRC Centre for Doctoral Training in Cloud Computing for Big Data, Newcastle University.

J. Shi - School of Mathematics and Statistics, Newcastle University L. Rochester \& S. Del Din* are with are with the Institute of Neuroscience | Newcastle University Institute of Ageing, Newcastle University, Newcastle upon Tyne, UK. L. Rochester is also with the Newcastle upon Tyne Hospitals NHS Foundation Trust, Newcastle upon Tyne, UK. (*phone:+441912081244; fax:+441912081251; emails: m.dunne-willows@ncl.ac.uk; p.watson@ncl.ac.uk; jian.shi@ncl.ac.uk; lynn.rochester@ncl.ac.uk; silvia.deldin@ncl.ac.uk*).
}

interest in new means of accurate objective clinical assessment of motor and gait impairments. This led to significant research interest in gait analysis for tracking disease progression and assessing medication efficacy [1].

Typically, gait analysis is performed using expensive and large laboratory systems such as pressure-sensor walkways. Advances in wearable technology have led to much smaller devices being commercially available and with greatly increased battery lives to the degree that prolonged accelerometry-based analyses are now practical [2]. Due to the complexity of gait and high clinical interest there have been a plethora of features have been suggested for gait analysis in the literature pertaining to several groups such as: spatio-temporal (ST), frequency domain as well as entropy measures to detect medication side effects (e.g. dyskinesia's) [3]. A subset of these has been proposed and validated by the BAM group as a comprehensive model of gait [4] which comprising five factors: pace, rhythm, asymmetry, variability and postural control, each with 3-4 features e.g. mean step velocity, step length variability etc. Novel gait features typically require scripted tasks for valid extraction or are associated with a minimum epoch of recording [5]. Both requirement put limitations the utility of their respective features and restrict their use in free-living environments.

Many features relating to the gait cycle (Fig. 1(a)) and have been identified as reliable biomarkers for PD [9, 7]. The asymmetric features of gait are of particular clinical interest in studies such as this as the asymmetric nature of PD, specifically the degree thereof, can be taken as a good biomarker for disease progression. In this context, phase plots offer an excellent means of gait assessment because they capture gait asymmetry (e.g. component angle $\alpha$ ) from a very short bout of gait / quiet walking. I.e. No unreasonable minimum epoch or scripted tasks are required to elicit a comprehensive phase plot [8].

In this piece, we present a means of exploiting the cyclic nature of gait to develop a compact fingerprint of a subject's gait using phase plot analysis. This will allow for tracking of changes in the disease through monitoring of the subtle changes in locomotion. Most importantly, this will be achievable on a per patient basis. The presented phase plot analysis offers a very compact means of assessing explaining deviations of PD gait from regular harmonic and sinusoidal motions in the context of disease state.

The aims of this work were therefore to (i) demonstrate the potential of phase plots as a sensitive tool to discriminate pathology (PD vs control group); (ii) explore the validity of 
phase plots for presenting a compact and comprehensive overview of a person's gait; and (iii) to assess the relation between features of phase plots to known/ gold-standard ST features of gait.

\section{METHODS}

\section{A. Participants}

41 PD patients within 4 months of diagnosis and 38 healthy aged matched control subjects (CL) were recruited from the Incidence of Cognitive Impairment in Cohorts with Longitudinal Evaluation-GAIT (ICICLE-GAIT) study. This is a collaborative study with ICICLE-PD, an incident cohort study (Incidence of Cognitive Impairment in Cohorts with Longitudinal Evaluation-Parkinson's disease) conducted between June 2009 and December. This study was conducted according to the declaration of Helsinki and had ethical approval from the Newcastle and North Tyneside research ethics committee. All participants signed an informed consent form prior to testing.

\section{B. Equipment and protocol}

Each participant was asked to wear a low-cost tri-axial accelerometer-based wearable device (Axivity AX3, 100Hz, $\pm 8 \mathrm{~g}$ ) located on the fifth lumbar vertebrae (L5). Participants were asked to walk at their preferred speed, performing four intermittent straight-line walking trials over $10 \mathrm{~m}$ walkway; a $7 \mathrm{~m}$ instrumented walkway (GAITRite/ gold standard) was placed in the centre to ensure gait was captured at a steady speed. PD participants who were on medication were tested approximately 1 hour after medication intake.

\section{Data Analysis}

The data were downloaded to a computer, segmented into the four different straight-line passes using time stamps and analysed by the MATLAB ${ }^{\circledR}$ program. Accelerometer signals were transformed to a horizontal-vertical coordinate system and filtered with a low-pass fourth order Butterworth filter at $20 \mathrm{~Hz}$.

\section{Algorithms}

Phase Plot construction: For consistency, we define the three accelerometer signals as $x_{1}, x_{2}$ and $x_{3}$ representing the medio-lateral, anterior-posterior, and longitudinal (vertical) axis respectively. Many gait analysis studies include some form of bout detection as a pre-processing stage before individual steps and gait events such as initial contacts (ICs) are extracted. Such methods typically incorporate information all three orthogonal axes. However, the labbased data was annotated with bout beginning and end times thus simplifying the process.

Step detection was carried out using methods outlined by Del Din et al [9] based on peak detection of the filtered vertical acceleration signal. Vertical excursion of the centre of mass was calculated using double integration methods similar to those described by Esser et al [8]. However, here numerical (a)

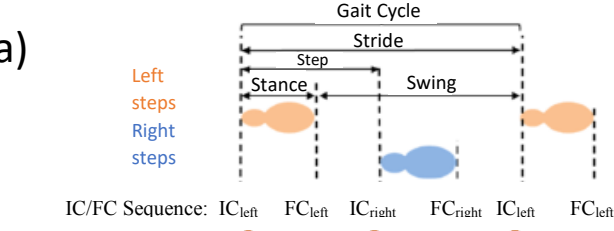

(b)

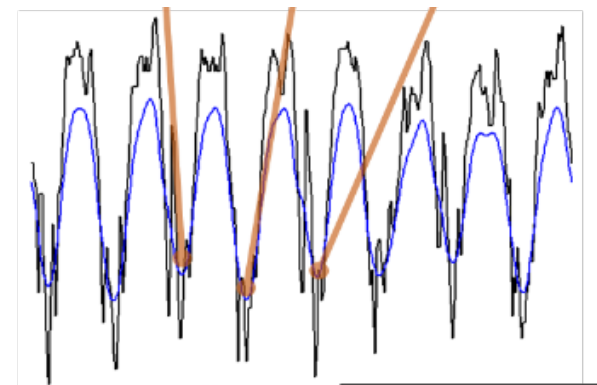

(c)

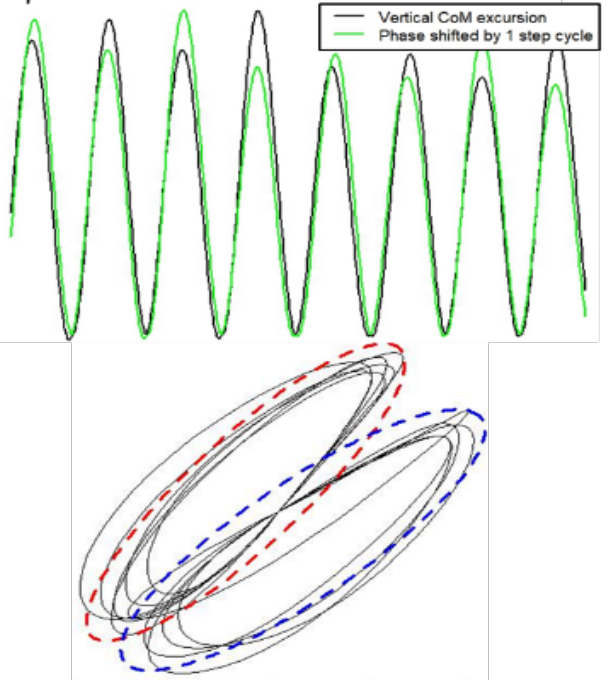

Figure 1. (a) Gait cycle. (b) Raw vertical acceleration (black) \& Butterworth filtered (blue). (c) Vertical CoM (centre of mass) excursion: original (black) and Phase shifted one step cycle (green). (d) Resultant phase plot with fitted Mixture bivariate Normal (Blue/Red).

integration was carried out piece-wise on IC-to-IC intervals of the vertical acceleration signal and included a detrending step to the intermediate velocity signal. Finally, concatenation $\&$ phase shifting. Following piecewise integration, the full vertical excursion signal must be restored via concatenation of the resultant integrals. Here the phase shift is introduced. We restore two such vertical excursion signals one of which is exactly one step cycle lagged behind the other i.e. $P_{1}(t)=$ $P_{0}(t-n)$ where $n$ is the number of data points comprising a step interval in the vertical excursion signal and $P_{1}$ and $P_{0}$ are the lagged and original vertical excursion signal respectively.

\section{E. Statistical analysis}

Phase plots generally take the form of two clusters of ellipses. This led us to approximate them according to a multivariate Gaussian mixture model i.e. the weighted sum of two bivariate Gaussian densities. In the first instance, we can classify the phase plots according to which order of as a Gaussian mixture model best approximates the data therein. 
Most plots can be approximated a Gaussian mixture model of order one or two, (Fig. 3D) i.e. they can be generally represented as one or two clusters of ellipses. One subject (excluded from study) produced an order three-phase plot, likely stemming from an atypical change in walking style during the short bout and was excluded from further analysis. Phase plots of order two are further classified into one of three subtypes each distinguishable by the relative form the two Gaussian components. Each type is intuitively named (Fig. 3): Single line (SL), Parallel wings (PW), Oblique wings (OW) and Thin line double (TLD). Thresholding values of $\alpha$ $=20^{\circ}, D=1.3$, and $E V R=2$, where $E V R$ is the ratio of the major and minor component ellipse axes, were chosen to discriminate most accurately between each of the four proposed plot types based on the defining features of each e.g. $\alpha$ chosen to ensure clear divergence of component directions.

Phase plots are constructible from a relatively small data sample- approximately 10 gait cycles (Fig. 1(a)). We have observed very high consistency within multiple phase plots of a given patient's gait. This reproducibility paired with the low data requirement (approximately 15-20 steps' worth of accelerometry data) means this method is highly generalisable. Another benefit of this method is that only a single axis of acceleration is required (vertical). Although, popular methods of bout extraction and other necessary preprocessing steps often require other axes' data. For example, Lyons et al [9] used pooled (three axis) SD of the signal to detect periods of motion.

To explore the physical interpretation of different phase plot types, the following five ST features, were evaluated for all PD subjects Step length, Step length asymmetry, step time variability, step velocity, and step velocity variability SD.

\section{RESULTS}

Table I. Participant Demographics and Characteristics

\begin{tabular}{|c|c|c|c|}
\hline Characteristic & PD (N=41) & CL (N=38) & $\boldsymbol{P}$ \\
\hline Age (years) & $66.7 \pm 9.7$ & $68.1 \pm 7.3$ & 0.23 \\
\hline Gender (M/F) & $13 / 28$ & $18 / 20$ & $<0.05$ \\
\hline $\begin{array}{c}\text { Hoehn \&Yahr } \\
\text { Stage (H-Y) }\end{array}$ & $\begin{array}{c}\mathrm{I}(12), \mathrm{II}(27), \\
\mathrm{III}(2)\end{array}$ & - & - \\
\hline BMI $\left(\mathrm{Kg} / \mathrm{m}^{2}\right)$ & $28.4 \pm 5.03$ & $28.17 \pm 4.18$ & 0.44 \\
\hline
\end{tabular}

Participant demographic, clinical and cognitive descriptors are shown in Table I. Compared to CL, PD Participants were aged matched; included proportionally more women (CL: 90\%, PD: 46.4\%); no differences were found between groups for BMI. Participants with PD were in the early stages of the disease with mild motor symptoms.

PD and CL subjects' show significantly different tendencies in all three defining features of given in figure 2 $(\mathrm{p}<0.05)$. Very high within subject consistency was observed $(\mathrm{p}<0.001)$ - for a given subject, their three phase plots would generally be very similar in type (Fig. 3) but also in terms of finer details. Indeed, there appeared to be great deal of unique detail in each subjects' phase plot. Consistency of plot type production was assessed after the classification shown in (Fig. 2) by comparison with randomly assigned types. The distribution of Types among both cohorts' phase plots is also significantly different. CL subject generally produce single line (SL) type plots $(90 \%)$. We found that several combinations (pairings) of plot types which represent significant changes in specific SP characteristics (Table II).

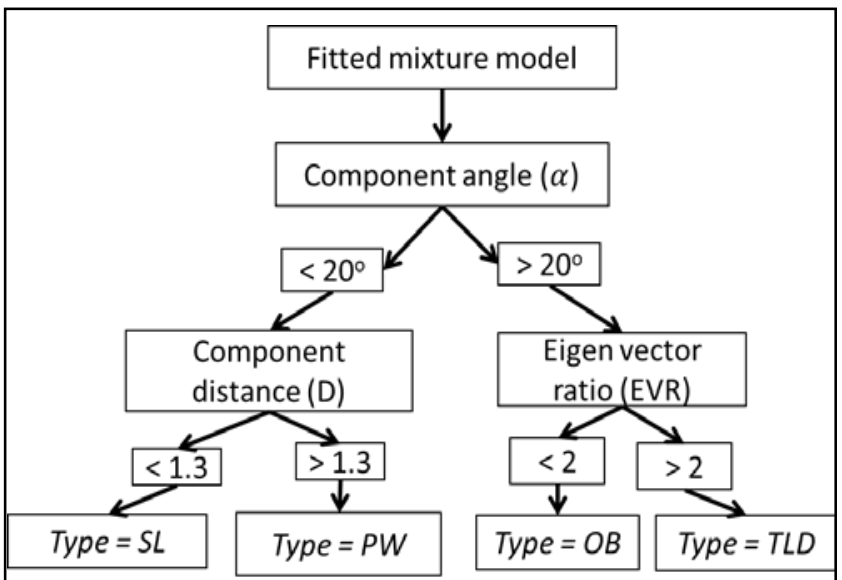

Figure 2. Phase plot classification algorithm. $\boldsymbol{\alpha}$ is the angle between both components, D is the Euclidean distance between both component centroids, EVR is the ratio of the major and minor component ellipse axes.

Table II. Spatio-temporal features for PD subjects. $\dagger$ most significant two pvalues of the six possible pair comparisons.

\begin{tabular}{|c|c|c|c|c|c|}
\hline $\begin{array}{c}\text { Type/ } \\
\text { feature }\end{array}$ & SL & TDL & PW & OW & $\mathrm{p}^{\dagger}$ \\
\hline $\begin{array}{c}\text { Step } \\
\text { Length (m) }\end{array}$ & $\begin{array}{c}0.636 \\
\pm 0.080\end{array}$ & $\begin{array}{c}0.552 \\
\pm 0.122\end{array}$ & $\begin{array}{c}0.721 \\
\pm 0.026\end{array}$ & $\begin{array}{c}0.729 \\
\pm 0.056\end{array}$ & $\begin{array}{c}\text { TDL vs PW } \\
\mathbf{0 . 0 2} \\
\text { SL vs PW } \\
\mathbf{0 . 0 4 7}\end{array}$ \\
\hline $\begin{array}{c}\text { Step } \\
\text { Length } \\
\text { Asymmetry } \\
(\mathrm{m})\end{array}$ & $\begin{array}{l}0.0275 \\
\pm 0.023\end{array}$ & $\begin{array}{l}0.0275 \\
\pm 0.021\end{array}$ & $\begin{array}{c}0.0405 \\
\pm 0.02\end{array}$ & $\begin{array}{c}0.0597 \\
\pm 0.01\end{array}$ & $\begin{array}{c}\text { TDL vs OW } \\
0.128 \\
\text { SL vs OW } \\
0.186\end{array}$ \\
\hline $\begin{array}{l}\text { Step Time } \\
\text { Variability } \\
\text { (s) }\end{array}$ & $\begin{array}{l}0.0177 \\
\pm 0.005\end{array}$ & $\begin{array}{l}0.0202 \\
\pm 0.006\end{array}$ & $\begin{array}{l}0.0115 \\
\pm 0.002\end{array}$ & $\begin{array}{l}0.0149 \\
\pm 0.004\end{array}$ & $\begin{array}{c}\text { SL vs PW } \\
0.063 \\
\text { TDL vs PW } \\
\mathbf{0 . 0 2 6}\end{array}$ \\
\hline $\begin{array}{c}\text { Step } \\
\text { Velocity } \\
\left(\mathrm{ms}^{-2}\right)\end{array}$ & $\begin{array}{c}1.16 \\
\pm 0.195\end{array}$ & $\begin{array}{c}0.974 \\
\pm 0.249\end{array}$ & $\begin{array}{c}1.42 \\
\pm 0.071\end{array}$ & $\begin{array}{c}1.52 \\
\pm 0.138\end{array}$ & $\begin{array}{c}\text { TDL vs PW } \\
\mathbf{0 . 0 0 7 9} \\
\text { SL vs PW } \\
\mathbf{0 . 0 3 8 8} \\
\end{array}$ \\
\hline $\begin{array}{c}\text { Step } \\
\text { Velocity } \\
\text { Var SD } \\
\left(\mathrm{m}^{2} \mathrm{~s}^{-4}\right)\end{array}$ & $\begin{array}{c}0.063 \\
\pm 0.024 \\
2\end{array}$ & $\begin{array}{c}0.074 \\
\pm 0.022\end{array}$ & $\begin{array}{c}0.085 \\
\pm 0.056\end{array}$ & $\begin{array}{l}0.0701 \\
\pm 0.017\end{array}$ & $\begin{array}{c}\text { SL vs TDL } \\
0.316 \\
\text { SL vs OW } \\
0.664\end{array}$ \\
\hline
\end{tabular}

\section{DISCUSSION \& CONCLUSION}

It is well established that sensor-based gait assessment is a reliable avenue [9] for PD monitoring, particularly in home environments. Pairing this with the ability of phase plot analysis to produce a comprehensive snapshot of gait, we present the possibility of fine-scale monitoring of PD progression and in the home environment.

Phase plot analysis satisfies key requirements for widescale deployment and clinical assessment: 1) results are objective, 2) Derivable from a single discreetly worn device and 3) very low demand on time and data- it can be implemented on a relatively small data set. We deduce from table II that a transition from TDL to PW correlates to significant increases in both step length and velocity which 


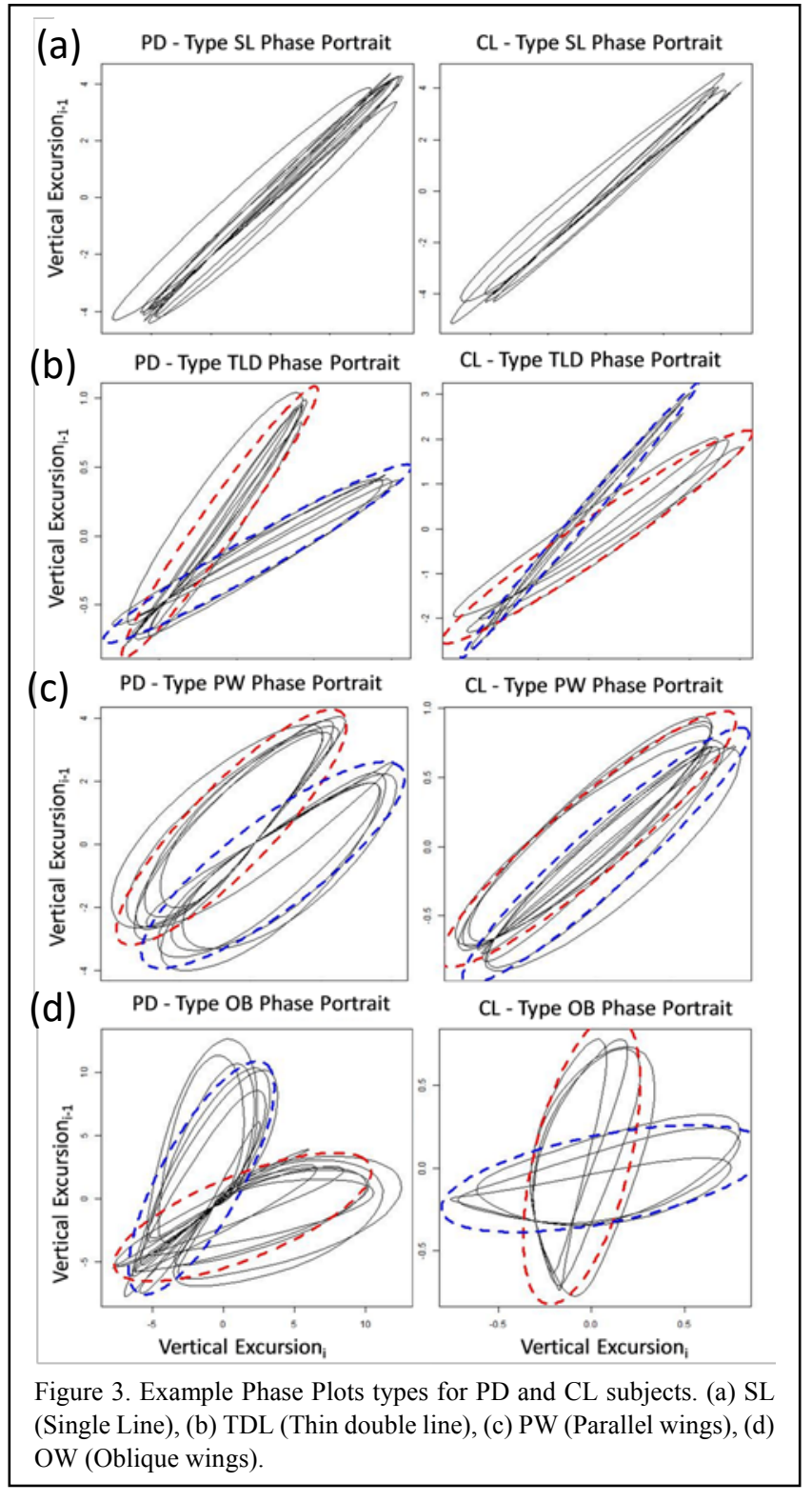

TABLE III. Mixture Model Derived Features and Classifications.

\begin{tabular}{|c|c|c|c|}
\hline & PD & CL & $p$ \\
\hline No. bouts & 117 & 100 & \\
\hline $\begin{array}{c}\text { Component } \\
\text { angle, } \alpha\left({ }^{\circ}\right)\end{array}$ & $12.9^{\circ} \pm 12.1^{\circ}$ & $8.9^{\circ} \pm 11.4^{\circ}$ & $\mathbf{0 . 0 1 4}$ \\
\hline Area ratio & $1.21 \pm 0.22$ & $1.34 \pm 0.37$ & $\mathbf{0 . 0 0 1 8}$ \\
\hline $\begin{array}{c}\text { Component } \\
\text { distance (D) }\end{array}$ & $0.847 \pm 1.16$ & $0.45 \pm 0.41$ & $\mathbf{0 . 0 0 0 7}$ \\
\hline Type - SL & 80 & 89 & $\mathbf{0 . 0 0 0 2}$ \\
\hline Type - PW & 11 & 3 & 0.093 \\
\hline Type - TDL & 23 & 7 & $\mathbf{0 . 0 0 9 5}$ \\
\hline Type - OB & 3 & 1 & 0.63 \\
\hline Order - 2 & 37 & 11 & $\mathbf{0 . 0 0 0 2}$ \\
\hline
\end{tabular}

reliably measure physical capability [9]. One might intuitively hypothesise that transitions to SL (most common subject type) would represent positive changes in ST characteristics. However, our results imply that walking with such a gait pattern as to produce a PW type phase plot as opposed to SL can result in improved gait parameters for PD subjects.

We showed that PD and CL subjects have significantly different tendencies in all three defining features of phase plots, this supports the utility of phase plot analysis as a tool sensitive to pathology (PD vs CL) and as a potential supplementary diagnostic tool.

The high within-subject consistency of phase plots features means that phase plots features can represent a compact and comprehensive overview of a person's gait. This leads us to adopt the fingerprint interpretation of phase plots to reflect their specificity not only to a given subject, but also to the state of their condition (PD in this case) at the time of recording.

Strong relationships between features of phase plots and ST features of gait were found. We identified several transitions of phase plot types which represent significant changes in specific ST characteristics, thus helping the interpretation of phase plot features by linking them to wellknown gait characteristics. Future work should aim to utilise other axes of acceleration to producing higher dimensional phase plots while taking care not to impede the interpretability of the output. Connections between phase plot characteristics and PD phenotypes should also be investigated. Due to the form of the data, an elliptical parameterisation of phase plots may be appropriate.

\section{ACKNOWLEDGMENTS}

The ICICLE-GAIT study was supported by Parkinson's UK (J-0802, G-1301) and by the NIHR Newcastle Biomedical Research Centre. SDD is supported by the Newcastle Biomedical Research Centre (BRC) based at Newcastle upon Tyne and Newcastle University. The work was also supported by the NIHR/Wellcome Trust Clinical Research Facility (CRF) infrastructure at Newcastle upon Tyne Hospitals NHS Foundation Trust. All opinions are those of the authors and not the funders.

\section{REFERENCES}

[1] T. Ilias, "Optimizing levodopa dosing routines for Parkinson's disease." PhD diss., Högskolan Dalarna, 2017.

[2] Ossig, "Wearable sensor-based objective assessment..." Journal of neural transmission 123, no. 1 (2016): 57-64.

[3] Godfrey, Medical Engineering \& Physics, vol. 30, pp. 1364-1386, 2008.

[4] Lord, "Independent domains of gait..." Journals of Gerontology Series A: Biomedical Sciences and Medical Sciences 68, no.7, 2012: 820-827.

[5] Weiss, "Toward automated, at-home assessment of mobility among patients with Parkinson disease, using a body-worn accelerometer." Neurorehabilitation and neural repair 25, no. 9 (2011): 810-818.

[6] S. Del Din, "Validation of an accelerometer to quantify a comprehensive battery of gait characteristics..." IEEE J Biomed Health Inform, Apr 2 2015.

[7] Palmerini, "Feature selection for accelerometer-based posture analysis in Parkinson's disease." IEEE Transactions on Information Technology in Biomedicine 15, no. 3 (2011): 481-490.

[8] P. Esser, "walking variability using phase plot analysis, Parkinson's disease," Gait Posture, vol. 38, pp. 648-52, Sep 2013.

[9] Lyons, G, "A description of an accelerometer-based mobility monitoring technique." Medical engineering \& physics 27, no. 6 (2005): 497-504

[10] Johansson, "Individualization of levodopa treatment using a microtablet dispenser and ambulatory accelerometry." CNS Neuroscience \& Therapeutics. 